\title{
Prevalência de demências no Brasil: um estudo de revisão sistemática
}

\author{
Prevalence of dementias in Brazil: a systematic review
}

\section{Prevalencia de demencias en Brasil: revisión sistemática}

\author{
Aline Laginestra-Silva1,2, Flávia Lúcia Gomes Tuyama ${ }^{1,2}$, Vera Regina \\ Cerceau ${ }^{1}$, Thiara Dias Café Alves Mariano ${ }^{1}$, Hudson Azevedo Pinheiro ${ }^{1}$, \\ Maria Liz Cunha de Oliveira ${ }^{2}$
}

1.Clínica especializada em Geriatria e Gerontologia, Brasilia-DF, Brasil.

2.Universidade Católica de Brasília, Brasilia-DF, Brasil.

\begin{abstract}
Resumo
Introdução. Trata-se de revisão sistemática realizada com estudos de prevalência para demências por fonte primária, considerando-se avaliação clínica e baterias multifuncionais em indivíduos nos diferentes estados brasileiros. Método. Foram elencados estudos dos últimos 10 anos, de 2008 a 2018. Resultados. Foram encontrados 17540 artigos em bases PubMed, Periódicos CAPES (Scielo, LILACS) e Google Acadêmico, sendo refinados para 500 estudos, excluindo-se os duplicados. As bases Cochrane Library e PROSPERO também foram consultadas, a fim de avaliar revisões já realizadas nas áreas, bem como em andamento. Estas não foram encontradas. Conclusão. Observou-se que as prevalências variaram de 4,9\% a $50 \%$, tendo maior representatividade da Região Sudeste. Os estudos encontrados revelaram que ainda contamos com pequena quantidade e baixa qualidade de pesquisas na área em foco, além destes estarem desatualizados.
\end{abstract}

Unitermos. Epidemiologia; Prevalência; Demência; Demência Brasil

\begin{abstract}
Introduction. This is a systematic review carried out with prevalence studies for dementia by primary source, considering clinical evaluation and multifunctional batteries in individuals in different Brazilian states. Method. Studies from the last 10 years, from 2008 to 2018, were listed. Results. 17540 articles were found in PubMed, CAPES journals (Scielo, LILACS), and Google Scholar, being refined to 500 studies, excluding duplicates. The Cochrane Library and PROSPERO databases were also consulted in order to assess revisions already carried out in the areas, as well as in progress. These were not found. Conclusion. It was observed that the prevalence ranged from $4.9 \%$ to $50 \%$, with greater representativeness in the Southeast Region. The studies found revealed that we still have a small quantity and low quality of research in the area in focus, in addition to being outdated.
\end{abstract}

Keywords. Epidemiology; Prevalence; Dementia; Dementia Brazil

\section{RESUMEN}

Introducción. Se trata de una revisión sistemática realizada con estudios de prevalencia de demencia por fuente primaria, considerando evaluación clínica y baterías multifuncionales en individuos de diferentes estados brasileños. Método. Se enumeraron los estudios de los últimos 10 años, de 2008 a 2018. Resultados. Se encontraron 17540 artículos en PubMed, revistas CAPES (Scielo, LILACS) y Google Scholar, siendo refinados a 500 estudios, excluyendo duplicados. También se consultaron las bases de datos de la Biblioteca Cochrane y PROSPERO, con el fin de evaluar las revisiones ya realizadas en las áreas, así como en curso. Estos no fueron encontrados. Conclusión. Se observó que la prevalencia osciló entre 4,9\% y 50\%, con mayor representatividad en la Región Sudeste. Los estudios encontrados revelaron que todavía tenemos una cantidad pequeña y de baja calidad de investigación en el área en cuestión, además de estar desactualizada.

Palabras clave: Epidemiología; Predominio; Demencia; Demencia Brasil 


\section{INTRODUÇÃO}

O termo demência vem do latim: de (significa falta) e mens (significa mente). Foi utilizado ao longo da história para caracterizar indivíduos que apresentavam deterioração de suas funções mentais cognitivas e comportamentais. 0 primeiro caso descrito de Doença de Alzheimer, causa mais comum de demência, foi feito por Alois Alzheimer, em 1906. Desde então, houve não só evolução das bases conceituais, como também mudanças epidemiológicas e diagnósticas ${ }^{1}$.

Neste contexto, entender a frequência com que síndromes demenciais aparecem em nosso meio, traçando sua incidência e prevalência, podem determinar fatores relacionados, implicando processos preventivos e tratamento mais eficaz².

Quanto a isso, estima-se que a prevalência de demências tenha dobrado nos últimos anos pelo mundo. Saber a especificidade de cada região, sobretudo em países em desenvolvimento, ajudará no planejamento de cuidados, políticas públicas, avaliação de custos e de seu impacto social ${ }^{3}$.

Trazendo estas informações para o Brasil, observa-se que o estudo da prevalência das demências é incipiente, 
concentrando-se em pequenas regiões, sem continuidade e sem informações abrangentes ${ }^{4}$.

Por toda especificidade e importância das demências, já se justifica uma revisão sistemática da literatura que possa sintetizar os achados científicos mais importantes, nos últimos dez anos, de forma a contribuir com direcionamentos futuros de novas investigações sobre a temática.

O objetivo deste estudo é resumir as evidências atuais sobre a prevalência de demência no Brasil, por fonte primária, analisando artigos publicados no período de 2008 a 2018, sistematizando as pesquisas na área.

\section{MÉTODO}

Esta revisão sistemática seguiu protocolo do Cochrane Handbook em sete passos definidos ${ }^{5}$, a saber: 1 - a formulação de uma pergunta de partida, como forma de guiar todo o processo; 2 - uma seleção de fontes para o estudo; 3 - uma triagem e análise crítica detalhada do material selecionado através de palavras-chave; 4 - análise e discussão das informações, conforme os objetivos propostos; 5 - interpretação dos dados em conformidade com os objetivos, esclarecendo os benefícios e aplicabilidade, os riscos e benefícios; 6 - atualização das informações de outras revisões, como contribuição estudos subsequentes sobre a temática; 7 - aprimoramento e atualização da revisão. 
Mais especificamente, a revisão centra-se na seguinte questão: Qual é a prevalência das demências no Brasil no período de 2008 a 2018 ?

Para localização dos artigos sobre diagnóstico de demência, inicialmente selecionou-se o material por títulos e por resumos, com as seguintes palavras-chave: Prevalence OR Epidemiology AND Dementia AND Brazil. A escolha dos bancos de dados eletrônicos justifica-se por se tratar dos mais acessíveis e conhecidos no Brasil. Utilizaram-se as seguintes plataformas: PubMed, Periódicos CAPES, Scielo, LILACS, Google acadêmico, Cochrane Library, PROSPERO. As duas últimas para avaliar revisões existentes e as já em andamento. Os artigos foram selecionados aleatoriamente, na medida em que apareciam na busca eletrônica, por palavras-chave.

Os critérios de inclusão utilizados foram: a) artigos completos; b) em português, espanhol ou inglês; c) com alguma das palavras-chave no título ou no resumo; d) estudos a partir de 2008; f) primários transversais ou de coorte prospectivos que apresentam diagnóstico das demências por avaliação clínica elou complementar. Os critérios de exclusão foram: a) estudos com datas anteriores a 2008; b) editoriais e/ou cartas ao editor. c) relatos de casos, d) estudos de revisão; e) estudos que não obtiveram confirmação das demências, que foram pouco relevantes, inconclusivos ou com abordagens específicas para determinado tipo de doença. 
A busca foi realizada por dois pesquisadores de forma independente, seguindo os critérios de inclusão e exclusão.

Os artigos selecionados foram analisados e registrados segundo esses itens: Nome do primeiro autor, ano de publicação, localização e duração do estudo, número de participantes, região (urbana ou rural), idade mínima e prevalência de demência. Qualquer discordância foi resolvida através da discussão com membros da equipe e pela escala Physiotherapy Evidence Database (PEDro) cuja estrutura foi organizada em cinco tópicos: Autor/Ano; Tema; Amostra; Método; Instrumento de Coleta de dados; Principais Resultados ${ }^{6}$.

Para a avaliação de viés, foram comparados os estudos por dados tabulados, verificando semelhanças e diferenças. A qualidade foi avaliada pelos componentes coletados descritos no item acima e confrontados ao padrão de referência adotado no projeto.

A validade externa foi avaliada pelo conhecimento da situação clínica e a interna pelo conhecimento do método científico. Utilizamos como método complementar Escala de Newcastle-Ottawa (NOS). Esta foi desenvolvida para avaliar a qualidade de estudos observacionais e contém oito itens que analisam três dimensões: seleção, comparabilidade e resultado (em caso de estudo de coorte) ou exposição (casocontrole) ${ }^{7}$.

Os estudos foram avaliados pela escala $\mathrm{NOS}^{7}$, sendo considerados com menor risco de viés aqueles com escore $\geq$ 3. Apenas um estudo apresentou alto risco de viés com 
escore 2. Para cada item, há uma série de opções em que aquela que reflete melhor a qualidade é pontuada por uma estrela, e quanto maior a quantidade de estrelas mais elevada é a qualidade do estudo.

\section{RESULTADOS}

Foram encontrados 17.540 estudos em todas as bases pesquisadas, sendo realizado refinamento por relevância e data para últimos 10 anos. Separados os duplicados, encontrados 500 artigos pelos moldes empregados. Destes, pelo título, foram afastados estudos de outros problemas que não o abordado e os de condições mórbidas específicas. Assim, foram selecionados 43 artigos.

Dos 43 artigos selecionados, 12 integraram a revisão, apresentando diagnóstico das demências por avaliação clínica com métodos de triagem semelhantes. Foram excluídos 31 artigos, 12 por serem de revisão, 8 deles por usarem métodos diferentes dos propostos, 9 por apresentarem objetivo do estudo diverso do selecionado, 1 por não ter sido encontrado na web, conforme descrito no fluxograma ilustrado na Figura 1.

Um total de 28.701 indivíduos foram investigados. Em 3 estudos com idade $\geq 50$ anos, em 8 estudos com $\geq 60$ anos e 1 estudo com $\geq 65$ anos. Muitos estudos não consideraram subtipos de demências, entretanto, os que fizeram diagnóstico diferencial, encontraram estatísticas de Demência de Alzheimer semelhante às encontradas 
mundialmente. Um estudo apenas encontrou taxa de prevalência bem maior que a habitual (50\%), por considerar avaliação neuropatológica.

Figura 1. Fluxograma de busca de dados.

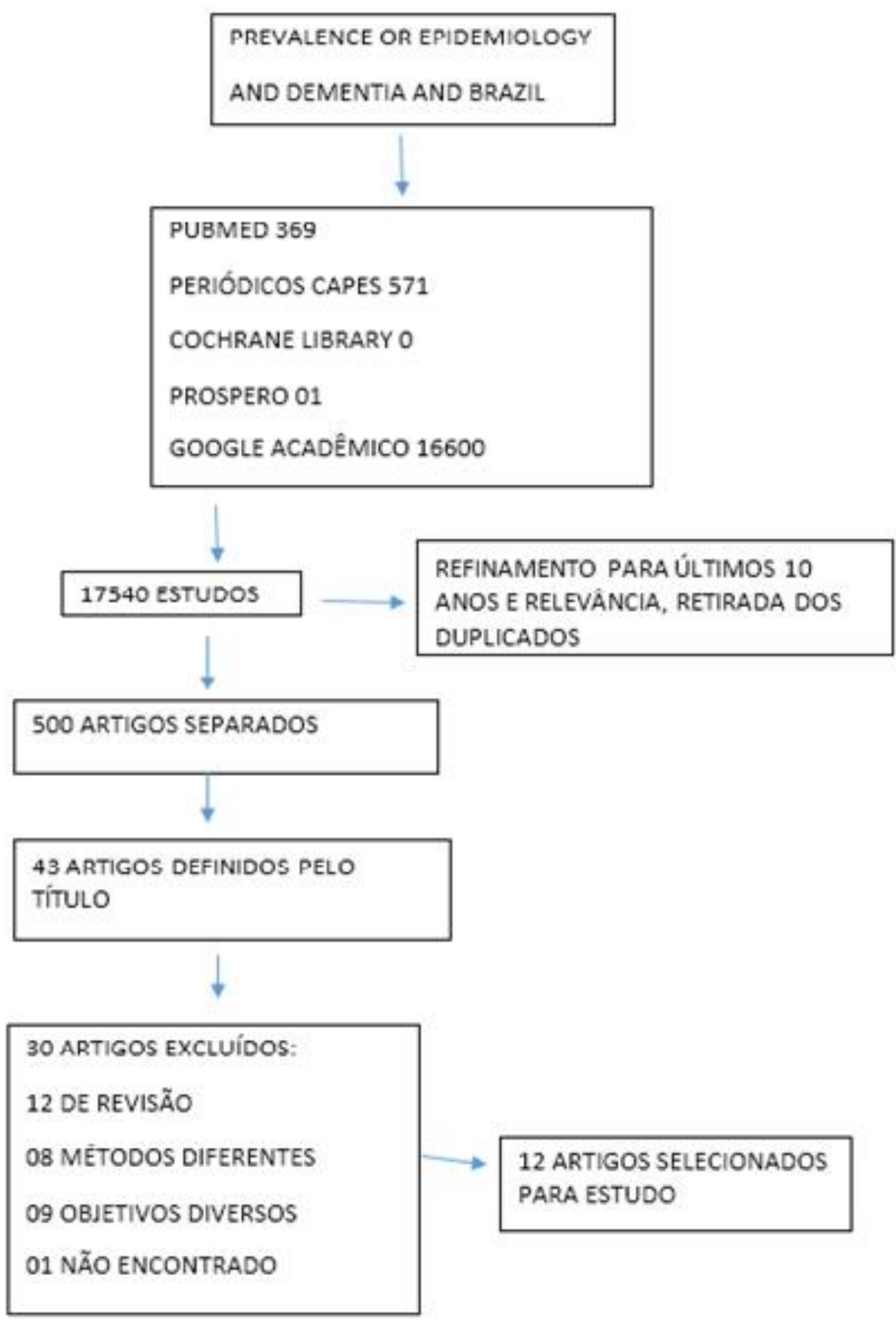


Todos os 12 estudos incluídos nesta revisão dividiram o método em fases e empregaram para avaliação clínica das demências baterias multifuncionais. 10 deles incluíram 0 Miniexame do Estado Mental (MEEM) de Folstein ${ }^{8}$ e todos utilizaram a escala de Estadiamento clínico das Demências $(C D R)^{9}$, levaram em conta histórico clínico, exames complementares, diferenças por sexo, idade e escolaridade. Os critérios diagnósticos foram do DSM-IV ${ }^{10}$. Apenas dois fizeram avaliações pós-morte e utilizaram o padrão ouro anatomopatológico.

Os dois estudos que apresentaram maior número de indivíduos foram os que fizeram avaliação em bancos de cérebros de serviços de verificação de óbito (SVO).

A prevalência da demência variou de $4,9 \%$ a $50 \%$, dependendo da região estudada e método empregado. Em todos os estudos, observou-se aumento com a idade e sexo masculino e baixa escolaridade, com exceção de um estudo que obteve muito baixa escolaridade e baixa prevalência. Deve-se levar em conta que os estudos em tela consideraram diferentes estádios de demência, não existindo padrão entre eles, conforme exposto na Tabela 1.

\section{DISCUSSÃO}

Os artigos analisados permitiram apontar a situação da prevalência de demência no Brasil em um período de dez anos, o que contribui para verificar as condições dessa doença no país. 
Tabela 1. Prevalência de demências em estudos brasileiros.

\begin{tabular}{|c|c|c|c|c|c|}
\hline Estudo & Localização & $\begin{array}{l}\text { Número de } \\
\text { participantes }\end{array}$ & $\begin{array}{l}\text { Prevalência } \\
(\%)\end{array}$ & Região & $\begin{array}{l}\text { Escore } \\
\text { de } \\
\text { qualidade }\end{array}$ \\
\hline Gondim et al ${ }^{10}$ & Fortaleza & 418 & 13,64 & Urbana & 3 \\
\hline Suemoto et al ${ }^{11}$ & São Paulo & 1.092 & 50 & Urbana & 5 \\
\hline César et $a^{12}$ & São Paulo & 630 & 17,5 & $\begin{array}{l}\text { Urbana e } \\
\text { Rural }\end{array}$ & 4 \\
\hline Maia et $a^{13}$ & Santa Catarina & 224 & 17,2 & Urbana & 3 \\
\hline Brucki et al ${ }^{14}$ & Amazonas & 163 & 4,9 & Rural & 3 \\
\hline Ferreira et $\mathrm{al}^{15}$ & Brasília & 60 & 30 & Urbana & 2 \\
\hline Grinber et $\mathrm{al}^{16}$ & São Paulo & 23.473 & 14,6 & Urbana & 5 \\
\hline Ribeiro et al ${ }^{17}$ & Rio de Janeiro & 683 & 16,9 & Urbana & 4 \\
\hline Lopes et $\mathrm{al}^{18}$ & São Paulo & 1.145 & 12,5 & Urbana & 3 \\
\hline Miranda et al ${ }^{19}$ & Minas Gerais & 218 & 6,4 & Urbana & 3 \\
\hline Chaves et al ${ }^{20}$ & Porto Alegre & 345 & 14,8 & Urbana & 3 \\
\hline Bottino et $\mathrm{al}^{21}$ & São Paulo & 250 & 6,8 & Urbana & 4 \\
\hline
\end{tabular}

Apesar das taxas de prevalência apresentadas pela literatura referirem-se a apenas oito áreas brasileiras, podese observar grandes diferenças entre elas, o que reflete diferenças no diagnóstico, nos métodos e nos processos de coleta de dados de cada estudo, que ocorreram em diferentes momentos ${ }^{13}$.

As áreas estudadas concentram-se na região Sudeste nos estados de São Paulo, Minas Gerais e Rio de Janeiro, tendo baixíssima representatividade na Região Sul, Norte e Centro-Oeste. Excluindo-se a cidade de São Paulo, cujo número de indivíduos foi extenso por considerar um SVO, as demais cidades tiveram pouquíssima expressividade, não podendo inferir dados para toda população do território.

O presente trabalho revisou diferentes estudos de prevalência no Brasil, em variadas regiões, apenas em 
artigos cuja casuística foi avaliação da demência por fonte primária, tendo em vista metodologias semelhantes que consideraram exame clínico através de baterias multifuncionais, sendo na maioria deles o MEEM utilizado como triagem cognitiva e todos com uso de CDR. Apenas dois estudos, além da avaliação clínica (histórico, CDR e outras baterias), complementaram o diagnóstico com exame anatomopatológico ${ }^{11,16}$.

Tais avaliações fazem crer que as demências ainda não mereceram atenção importante dos pesquisadores brasileiros, bem como de serviços públicos ou privados de saúde, não representando prioridade, muito embora o impacto dessas doenças seja de grande importância sociofamiliar.

É importante salientar que o diagnóstico das demências, principalmente a Demência de Alzheimer, na prática clínica, define-se, no máximo, como possibilidade ou probabilidade; somente o anatomopatológico seria definitivo de acordo com estudos atuais. Tais considerações, justificam a discrepância em prevalência de pesquisas que abordam como critério diagnóstico o estudo direto do tecido cerebral e suas disfunções, uma vez que os sinais e sintomas relacionados podem ser inespecíficos e com intersecções em diversas condições nosológicas ${ }^{22}$.

Considera-se, ainda, a tentativa de avanços em estudos que tentam admitir um construto biológico in vivo para melhor acurácia diagnóstica, buscando um resultado definitivo em meio a achados clínicos muito semelhantes a 
diversas causas. Foi definido a neuroimagem para comprovar depósito amiloide, proteína tau, bem como neurodegeneração, embora o uso na prática não esteja acessível, sobretudo em países pobres ${ }^{23}$.

Foi considerado o impacto de biomarcadores para relevância diagnóstica, entretanto, em amostra sanguínea, mostrou-se inconsistente. Desta forma, os resultados encontrados em estudos de prevalência, mostram-se, muitas vezes conflitantes e discrepantes, conforme o método utilizado ${ }^{24}$.

Destacam-se como limitações: pouco tempo estabelecido para pesquisa, baixa qualidade dos estudos encontrados, diversidade dos métodos empregados, dificultando comparação entre eles, presença de objetivos variados que não eleitos para a presente revisão.

\section{CONCLUSÕES}

O objetivo deste trabalho foi a obtenção de uma estimativa do crescimento de idosos demenciados no futuro próximo. Espera-se, com isso, subsidiar o processo de formulação de políticas na área de saúde pública. Os resultados apontaram para um crescimento desse segmento, dado o aumento esperado da população muito idosa, ainda que a prevalência não cresça. Chamam a atenção para uma nova e urgente questão ética: o cuidado necessário a ser dispensado às pessoas idosas com demência. 
Conclui-se ser inegável o baixo número de pesquisas sobre prevalência de demências no Brasil, sendo os trabalhos na área pequenos e desatualizados.

Espera-se, a partir do presente estudo, fomentar e estimular a elaboração de novas pesquisas abordando efetivamente o diagnóstico das demências em nosso país.

\section{REFERÊNCIAS}

1.Pessoa RMP, de Faria SM, de Morais D, Chagas MHN. Da Demência ao Transtorno Neurocognitivo Maior: Aspectos Atuais. Cienc Saude 2016;6:5-17. https://doi.org/10.21876/rcsfmit.v6i4.606

2.National Academies of Sciences, Engineering, and Medicine; Health and Medicine Division; Board on Health Sciences Policy; Committee on Preventing Dementia and Cognitive Impairment; Alan I. Leshner, Story Landis, Clare Stroud, and Autumn Downey (Eds). Preventing cognitive decline and dementia: A way forward. Consensus Study Report. Washington (DC): National Academies Press. 2017. https://doi.org/10.17226/24782

3. Krysinska K, Sachdev PS, Breitner J, Kivipelto M, Kukull W, Brodaty $\mathrm{H}$. Dementia registries around the globe and their applications: $A$ systematic review. Alzheimer's Dement 2017;13:1031-47. https://doi.org/10.1016/j.jalz.2017.04.005

4.Burlá C, Camarano AA, Kanso S, Fernandes D, Nunes R. Panorama prospectivo das demências no Brasil: um enfoque demográfico. Cien Saude Colet 2013;18:2949-56.

http://dx.doi.org/10.1590/S1413-81232013001000019

5. Moher D, Liberati A, Tetzlaff J, Altman DG, PRISMA Group. Preferred reporting items for systematic reviews and meta-analyses: the PRISMA statement. PLoS Med 2009;6:e1000097.

http://dx.doi.org/10.1371/journal.pmed.1000097

6.Clarke M, Horton R. Bringing it all together: Lancet-Cochrane collaborate on systematic reviews. Lancet 2001;357:1728. http://dx.doi.org/10.1016/50140-6736(00)04934-5

7.Stang A. Critical evaluation of the Newcastle-Ottawa scale for the assessment of the quality of nonrandomized studies in meta-analyses. Eur J Epidemiol 2010;25:603-5. http://dx.doi.org/10.1007/s10654-010$9491-z$

8. Brito-Marques PR de, Cabral-Filho JE. The role of education in minimental state examination: a study in Northeast Brazil. Arq Neuropsiquiatr 2004;62:206-11.

http://dx.doi.org/10.1590/S0004-282X2004000200003

9. Maia ALG, Godinho C, Ferreira ED, Almeida V, Schuh A, Kaye J, et al. 
Application of the Brazilian version of the CDR scale in samples of dementia patients. Arq Neuropsiquiatr 2006;64:485-9.

http://dx.doi.org/10.1590/S0004-282X2006000300025.

10.Gonçalves-Pereira M, Cardoso A, Verdelho A, Silva JA, Almeida $M C$, Fernandes $A$, et al. The prevalence of dementia in a Portuguese community sample: a 10/66 Dementia Research Group study. BMC Geriatr 2017;17:261. https://doi.org/10.1186/s12877-017-0647-5

11.Suemoto CK, Ferretti-Rebustini REL, Rodriguez RD, Leite REP, Soterio L, Brucki SMD, et al. Neuropathological diagnoses and clinical correlates in older adults in Brazil: A cross-sectional study. PLoS Med 2017;14:e1002267.

https://doi.org/10.1371/journal.pmed.1002267

12.César KG, Brucki SMD, Takada LT, Nascimento LFC, Gomes CMS, Almeida MCS, et al. Prevalence of cognitive impairment without dementia and dementia in Tremembé, Brazil. Alzheimer Dis Assoc Disord 2016;30:264-71.

https://doi.org/10.1097/WAD.0000000000000122

13. Maia E, Steglich MS, Lima AP, Troncoso IHZ, Silva KI, Martins TRC, et al. Dementia in elderly inpatients admitted to medical wards in $B$ razil: diagnosis and comorbidity with other clinical diseases. Psychogeriatrics 2016;16:177-84. https://doi.org/10.1111/psyg.12136 14. Brucki SMD, Nitrini R. Cognitive impairment in individuals with low educational level and homogeneous sociocultural background. Dement Neuropsychol 2014;8:345-50.

http://dx.doi.org/10.1590/S1980-57642014DN84000007

15.Ferreira LS, Pinho M do SP, Pereira MW de M, Ferreira AP. Perfil cognitivo de idosos residentes em Instituições de Longa Permanência de Brasília-DF. Rev Bras Enferm 2014;67:247-51.

http://dx.doi.org/10.5935/0034-7167.20140033

16.Grinberg L, Nitrini R, Suemoto C, Ferretti-Rebustini REL, Leite REP, Farfel JM, et al. Prevalence of dementia subtypes in a developing country: a clinicopathological study. Clinics 2013;68:1140-5. https://doi.org/10.6061/clinics/2013(08)13

17.Ribeiro PCC, de Souza Lopes C, Lourenço RA. Prevalence of dementia in elderly clients of a private health care plan: a study of the FIBRA-RJ, Brazil. Dement Geriatr Cogn Disord 2013;35:77-86. https://doi.org/10.1159/000345984

18.Lopes MA, Ferrioli E, Nakano EY, Litvoc J, Bottino CMC. High prevalence of dementia in a community-based survey of older people from Brazil: association with intellectual activity rather than education. J Alzheimer's Dis 2012;32:307-16. https://doi.org/10.3233/JAD2012-120847

19.de Paula Miranda L, Silveira MF, Oliveira TL, Alves SFF, Júnior HM, Batista AUD, et al. Cognitive impairment, the Mini-Mental State Examination and socio-demographic and dental variables in the elderly in Brazil. Gerodontology 2012;29:e34-40.

https://doi.org/10.1111/j.1741-2358.2011.00541.x

20.Lorena Chaves M, Luiza Camozzato A, Godinho C, Piazenski I, Kaye J. Incidence of mild cognitive impairment and Alzheimer disease in 
Southern Brazil. J Geriatr Psychiatry Neurol 2009;22:181-7. https://doi.org/10.1177/0891988709332942

21. Bottino CMC, Azevedo Jr D, Tatsch M, Hototian SR, Moscoso MA, Folquitto J, et al. Estimate of dementia prevalence in a community sample from São Paulo, Brazil. Dement Geriatr Cogn Disord 2008;26:291-9. https://doi.org/10.1159/000161053

22.Association AP. DSM-5: Manual Diagnóstico e Estatístico de Transtornos Mentais. Porto Alegre: Artmed Editora; 2014.

23. Jack Jr CR, Bennett DA, Blennow K, Carrillo MC, Dunn B, Haeberlein $\mathrm{SB}$, et al. NIA-AA research framework: toward a biological definition of Alzheimer's disease. Alzheimer's Dement. 2018;14:535-62. https://doi.org/10.1016/j.jalz.2018.02.018

24.Pais M, Martinez L, Ribeiro O, Loureiro J, Fernandez R, Valiengo L, et al. Early diagnosis and treatment of Alzheimer's disease: new definitions and challenges. Brazilian J Psychiatry 2020;42:431-41. http://dx.doi.org/10.1590/1516-4446-2019-0735. 